\title{
Automated Switching Mechanism for Multi-Standard RFID Transponder
}

\author{
Teh Kim Ting and Khaw Mei Kum \\ Faculty of Engineering \\ Multimedia University \\ Cyberjaya, Malaysia \\ mkkhaw@mmu.edu.my
}

\author{
Faisal Mohd-Yasin \\ Queensland Micro- and Nanotechnology Centre \\ Griffith University \\ Brisbane, Australia \\ f.mohd-yasin@griffith.edu.au
}

\begin{abstract}
This paper presents an automated switching mechanism for a multi-standard modulator operating at high frequency (13.56 $\mathrm{MHz}$ ) passive Radio Frequency Identification (RFID) transponder. The design adheres to the automatic switching of ISO 14443 (with a data rate of $106 \mathrm{kbps}$ ), and ISO 15693 Single Subcarrier (with data rate of $6.62 \mathrm{kbps}$ and 26.48 kbps). The concept of the automated selection of the two different protocols is based on the incoming RF amplitudes; taking advantage of the different reading distances of the two protocols. The switch design is made up of envelope detector, CMOS operational amplifier, comparator with hysteresis, CMOS voltage reference and a 2-to-1 multiplexer. Simulation results verified that the proposed switch design is capable of switching to the desired ISO standard accordingly in a multi-standard transponder. This simulation work is designed and verified by

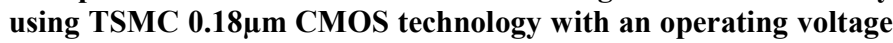
of $1.8 \mathrm{~V}$.
\end{abstract}

\section{INTRODUCTION}

In recent years, contactless Radio Frequency Identification (RFID) has proliferated in many service industries, purchasing and distribution logistics industries, manufacturing companies and material flow systems. This RFID technology uses electromagnetic waves for automatic identification (auto ID) of people or objects. The aim of most auto-ID systems is to increase flexibility and efficiency, reduce data entry errors, labor cost as well as prevent internal theft. In an RFID system, the transponder or tag is a data-carrying device integrated on the object to be tracked, whereas the reader or interrogator is a data capture device. Naturally, a multi-standard transponder will be an advantage for wider range of applications due to their flexible operational distances and transmission methods, while maintaining similar costs. From a technical paper discussing on the multi-standard compatible RFID transponder [1], the selectors chosen to decide on the type of standard used by the data transfer from the transponder to the reader were previously done manually.

The goal of this work is to provide an automated switching mechanism that selects the desired protocol between the transponder and the interrogator, in a multi-standard modulator working in $13.56 \mathrm{MHz}$ of a passive RFID transponder. Table 1 shows the summary and comparisons between the proximity standard of ISO 14443 and the vicinity standard of ISO 15693 Single Subcarrier. The major difference between both standards is that proximity cards need to be within $10 \mathrm{~cm}$ range while vicinity cards can be read within $50 \mathrm{~cm}$ range, up to $1 \mathrm{~m}$.
Therefore, the relationship between the recognition range of transponder to the reader and the voltage induced at the transponder are utilized for the automated switching concept. Particularly, wider distance between transponder and reader results in smaller amplitude of voltage received by the reader [3]-[4].

Based on a multi-standard $13.56 \mathrm{MHz}$ RFID system research paper, the measured induced voltage using ISO 14443 Type B commercial tag at a recognition distance of $10 \mathrm{~cm}$ is $2.644 \mathrm{~V}[2]$. Hence, this voltage is used as the reference voltage to switch between the ISO 14443 and ISO 15693 standards signals accordingly. The desired ISO standard protocol is selected automatically, in accordance with the comparison of the received RF input signal against the reference voltage using a comparator circuit.

TABLE I

Summary And Comparisons BetweEn Specifications Of ISO 14443 And ISO 15693 [1]-[2]

\begin{tabular}{|c|c|c|c|}
\hline \multirow[t]{2}{*}{ ISO Standard } & \multicolumn{2}{|c|}{ Proximity cards } & \multirow{2}{*}{$\begin{array}{l}\text { Vicinity card } \\
15693 \text { (Single } \\
\text { Subcarriers) }\end{array}$} \\
\hline & $14443 \mathrm{~A}$ & 14443B & \\
\hline $\begin{array}{l}\text { Operating } \\
\text { frequency, } \mathbf{f}_{\mathrm{c}}\end{array}$ & \multicolumn{3}{|c|}{$13.56 \mathrm{MHz} \pm 7 \mathrm{kHz}$} \\
\hline $\begin{array}{l}\text { Data Coding } \\
\text { (Reader to tag) }\end{array}$ & $\begin{array}{l}\text { ASK } 100 \%(\mathrm{OOK}) \\
\text { Modified Miller }\end{array}$ & $\begin{array}{l}\text { ASK 10\% } \\
\text { NRZ }\end{array}$ & $\begin{array}{l}\text { ASK } 10 \% \text { or } 100 \% \\
\text { PPM }\end{array}$ \\
\hline \multirow[t]{2}{*}{$\begin{array}{l}\text { Data Coding } \\
\text { (Tag to reader) }\end{array}$} & $\begin{array}{l}\text { 106kbps data rate: } \\
\text { ASK } 100 \% \text { (OOK) } \\
\text { Manchester }\end{array}$ & $\begin{array}{l}\text { l06kbps data rate: } \\
\text { BPSK } \\
\text { NRZ-L }\end{array}$ & \multirow[t]{2}{*}{$\begin{array}{l}\text { ASK } \\
\text { Manchester }\end{array}$} \\
\hline & \multicolumn{2}{|c|}{$\begin{array}{l}212 \text { kbps, } 424 \text { kbps, } 848 \text { kbps data rate: } \\
\text { BPSK } \\
\text { NRZ-L }\end{array}$} & \\
\hline $\begin{array}{l}\text { Subcarrier } \\
\text { frequency, } \mathrm{f} \text {. }\end{array}$ & \multicolumn{2}{|c|}{$847.5 \mathrm{kHz}$} & $423.75 \mathrm{kHz}$ \\
\hline \multirow{2}{*}{$\begin{array}{l}\text { Read or write } \\
\text { range }\end{array}$} & \multicolumn{2}{|c|}{ Up to $10 \mathrm{~cm}$} & Up to $1 \mathrm{~m}$ \\
\hline & $\sim 2 \mathrm{~cm}$ & $\sim 10 \mathrm{~cm}$ & $\sim 20 \mathrm{~cm}$ \\
\hline $\begin{array}{l}\text { Required } \\
\text { Bandwidth }\end{array}$ & \multicolumn{2}{|c|}{$1.7 \mathrm{MHz}$} & $1 \mathrm{MHz}$ \\
\hline $\begin{array}{l}\text { Q-factor of TX } \\
\text { antenna }\end{array}$ & \multicolumn{2}{|c|}{ Less than 8} & 13.6 \\
\hline$I_{\text {tims }}$ & $1 \mathrm{~mA}$ & $25 \mathrm{~mA}$ & $\sim 220 \mathrm{~mA}$ \\
\hline$V_{\text {pegle }}$ & $0.57 \mathrm{~V}$ & $2.16 \mathrm{~V}$ & $7.41 \mathrm{~V}$ \\
\hline$V_{\text {induced }}$ at tag & \multicolumn{2}{|c|}{$\begin{array}{l}\sim 2.644 \mathrm{~V} \text { (Based on 14443B at recognition } \\
\text { distance of } 10 \mathrm{~cm} \text { ) }\end{array}$} & $\begin{array}{l}1.6833 \mathrm{~V} \text { (reader } \\
\text { distance of } 50 \mathrm{~cm} \text { ) }\end{array}$ \\
\hline
\end{tabular}

The architecture of the automated switch design for passive RFID transponder is described in Section II, while Section III demonstrates the functions of each building block. Simulation results to validate the performance of the automated switch are shown in Section IV. Finally, the conclusion is stated in section $\mathrm{V}$. 


\section{CIRCUIT ARCHITECTURE}

Figure 1 shows the proposed block diagram of the automated switch design for the multi-standard HF passive RFID transponder. The automated switching concept is made up of envelope detector, CMOS operational amplifier, comparator with hysteresis, CMOS voltage reference and a 2to-1 multiplexer.

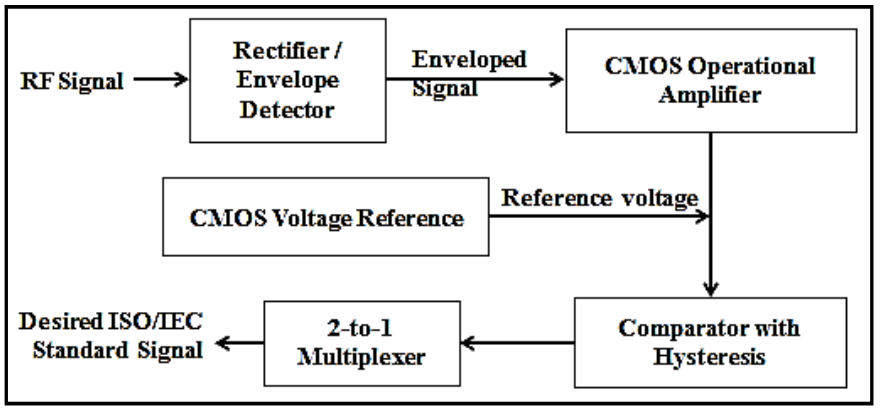

Fig. 1. Block diagram of an automated switch design for a multi-standard passive RFID transponder

According to the block diagram, the working principle of the switching mechanism commences with the detection and conversion of high frequency RF signal into the equivalent amplitude of enveloped signal. After the envelope detection, the amplitude of the enveloped RF signal is decreased. Therefore, a CMOS operational amplifier is required to provide the desired amount of amplification to the enveloped signal. In addition, some filtering effect which is capable of eliminating the high frequency ripple voltage contained in the enveloped signal is performed in this stage as well.

Subsequently, the amplified signal is fed into a comparator circuit. In particular, the comparator converts its input signal, $\mathrm{V}_{\text {in }}$ to a digital pulse of either a high $\left(\mathrm{V}_{\mathrm{DD}}\right)$ or a low (ground) output, in accordance with the reference voltage $\mathrm{V}_{\mathrm{REF}}$. In this switch design, a comparator with hysteresis is designed so that the circuit is immune to the high frequency noise and jitter. Moreover, in order to reduce the number of off-chip component, a comparator with internal hysteresis is designed.

To supply a constant reference voltage to the comparator, a CMOS voltage reference circuit is included. Since the desired standard is selected based on whether the input signal is greater or lesser than reference voltage, it is essential for the $V_{R E F}$ to be stable and independent of the temperature and supply voltage variations. Particularly, as $\mathrm{V}_{\text {in }}$ is greater than $\mathrm{V}_{\mathrm{REF}}$, low output signal will be produced and hence it will refer to ISO14443 standard. In contrast, if $\mathrm{V}_{\text {in }}$ is smaller than $\mathrm{V}_{\mathrm{REF}}$, then the high output signal will correspond to the standard of ISO15693.

The last part of the switch design is a 2-to-1 multiplexer. Figure 2 illustrates the proposed integration of switch design in the multi-standard RFID transponder protocol. By referring to Figure 2, the subcarrier signal of $847.5 \mathrm{kHz}$ which represents ISO14443 as well as subcarrier signal of $423.75 \mathrm{kHz}$ denotes
ISO15693 from the frequency divider will be utilized as the input signals, IN_0 and IN_1 of the 2-to-1 multiplexer respectively. Furthermore, the output of the comparator circuit acts as the selector signal of the multiplexer. Therefore, when selector signal is low, IN_0 is output, so ISO14443 signal will be selected automatically. On the contrary, IN_1 signal that is corresponds to ISO15693 standard will be produced when the selector signal is high.

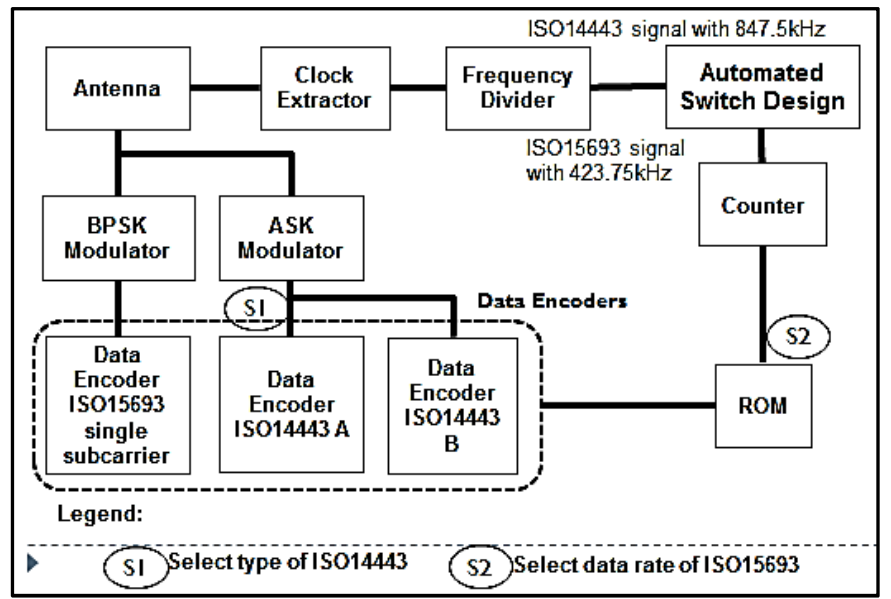

Fig. 2. Proposed integration of automated switch mechanism in multistandard RFID transponder protocol

\section{FUNCTIONAL BLOCKS}

\section{A. Complete Envelope Detector with Cascaded Doubler Cell}

The peak of the radio frequency input signal is track by an envelope detector circuit as output signal. Since the conventional envelope detector with diode-connected PMOS, capacitor and resistor is inappropriate to be used in a high frequency circuit design [5], the complete envelope detector with cascaded doubler cell as shown in Figure 3 is designed. As it is desirable for the comparison level to be at zero voltage, the cascaded doubler cell acts as the negative DC voltage which replaces the ground terminal [6].

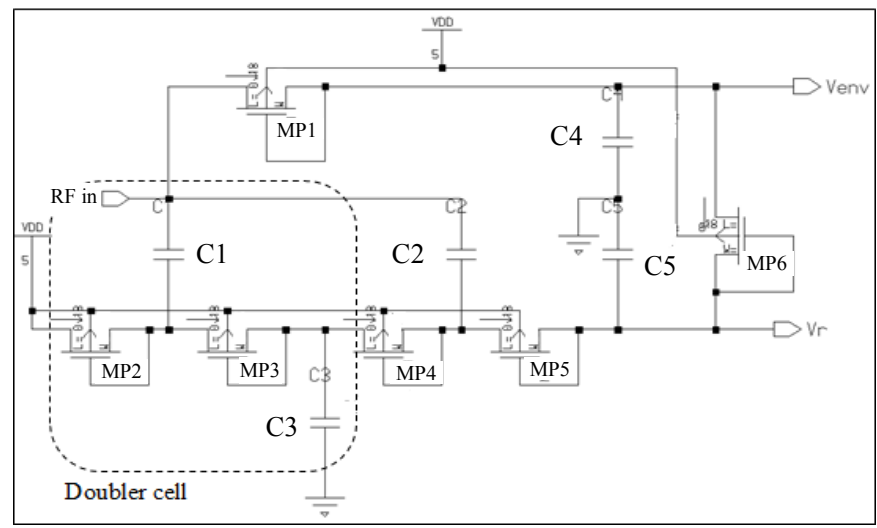

Fig. 3. Complete Envelope Detector Circuit 


\section{B. CMOS Operational Amplifier}

Figure 4 shows the actual configuration of a two-stage, internally compensated non-inverting CMOS operational amplifier (op-amp). Theoretically, this op-amp is capable of providing good voltage gain, good common-mode range and good output swing [7]. The two-stage op-amp comprises a differential stage and a gain stage. In the differential stage, differential amplification and differential to single-ended conversion are achieved, whereas additional gain is provided by the gain stage. A compensation capacitor, $\mathrm{C} 2$ is included between differential stage and gain stage to ensure the steadiness of op-amp for the feedback. Moreover, in case of a non-inverting op-amp, the gain can be defined as,

$$
\text { Voltage gain, } A=1+\frac{R_{2}}{R_{4}}
$$

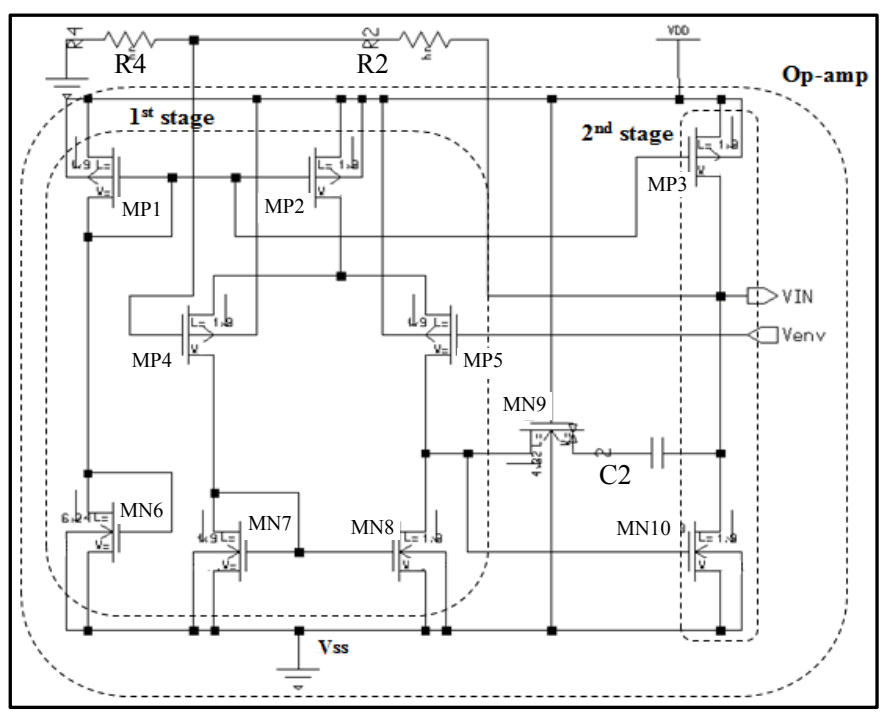

Fig. 4. CMOS Operational Amplifier

\section{Comparator with Internal Hysteresis}

The configuration of a comparator with internal hysteresis is shown in Figure 5. The hysteresis effect is provided by a latch structure comprising of MP2, MP3, MP4 and MP5. Besides, a bias circuit which composed of MN4 and a current source is included to supply a stable voltage to the gate of MN6. Particularly, larger value of current source will result in higher power dissipation but better output performance. Therefore, appropriate value of current source is required to compromise power consumption and circuit performance.

At the output stage, an inverter formed by MP6 and MN5 is utilized to output a binary signal, based on the comparison between $V_{\text {in }}$ and $V_{\text {REF. }}$. As $V_{\text {in }}$ is larger than $V_{\text {REF }}$, then MP6 is turned off while MN5 is turned on, hence output is pulled to logic low of $0 \mathrm{~V}$. Alternatively, when $\mathrm{V}_{\text {in }}$ is smaller than $\mathrm{V}_{\mathrm{REF}}$, MP6 is turned on and MN5 is turned off, thus, the output voltage is pulled up and fixed as $\mathrm{V}_{\mathrm{DD}}$ or $1.8 \mathrm{~V}$.

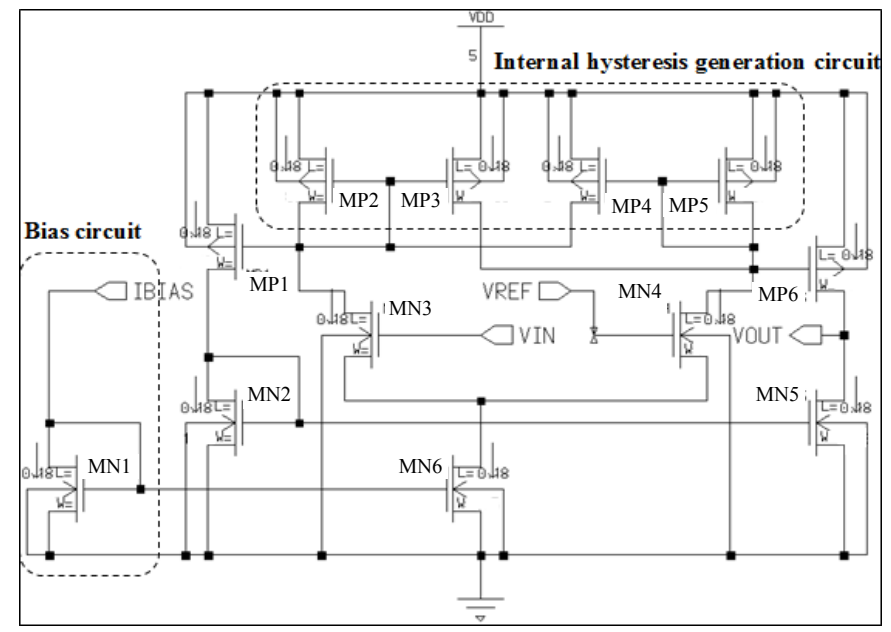

Fig. 5. Comparator with Internal Hysteresis

\section{CMOS Voltage Reference}

Figure 6 illustrates the configuration of a voltage reference circuit. Since the desired ISO standards are selected based on the comparison of the input signal with a reference voltage, it is essential for $V_{\mathrm{REF}}$ to be independent of temperature variation, power supply variation, load variation and other operating conditions. The output reference voltage can be defined as [8],

$$
\mathrm{V}_{\mathrm{REF}}=\frac{\mathrm{V}_{\mathrm{GS}}}{\frac{\mathrm{R}_{1}}{\mathrm{R}_{2}}+1}+\frac{\mathrm{V}_{\mathrm{GS} 8}}{\frac{\mathrm{R}_{2}}{\mathrm{R}_{1}}+1}
$$

According to the equation $2, \mathrm{~V}_{\mathrm{REF}}$ can be controlled by adjusting the ratio of $R_{1}$ and $R_{2}$ and thus the sensitivity of temperature coefficient of the output voltage against the change of $R_{1}$ and $R_{2}$ ratio is minimized. Besides, specific amplification through the implementation of an op-amp as well as voltage-controlled voltage source (VCVS) will be applied to the resulted reference voltage, in order to obtain final reference voltage of $2.644 \mathrm{~V}$ that is required by the comparator circuit.

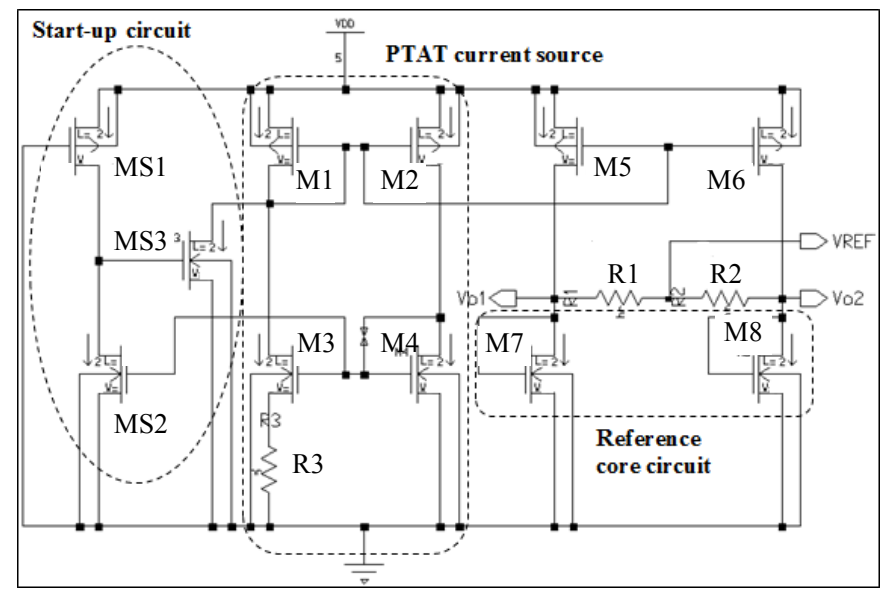

Fig. 6. CMOS Voltage Reference Circuit 


\section{E. 2-to-1Multiplexer}

A 2-to-1 multiplexer acts as a selector that selects between two available choices. The circuit of a pass-transistor based 2to-1 multiplexer is shown in Figure 7 . The pass transistors are capable of preventing threshold voltage loss, which will sequentially minimize the possibility of static power consumption [9]. As mentioned in Section II, the subcarrier signals for ISO 14443 and ISO 15693, which generated by the frequency divider of multi-standard RFID transponder protocol, are utilized as the input signals, IN_0 and IN_1 of multiplexer respectively.

In addition, the output signal from the comparator circuit is employed as the selector signal, SELECTOR and the output signal is denoted as MUX_OUT. When SELECTOR is low, IN 0 is output as MUX OUT, thus ISO14443 signal can be selected automatically. On the other hand, IN_1 signal that is corresponded to ISO15693 standard is produced when selector signal is high.

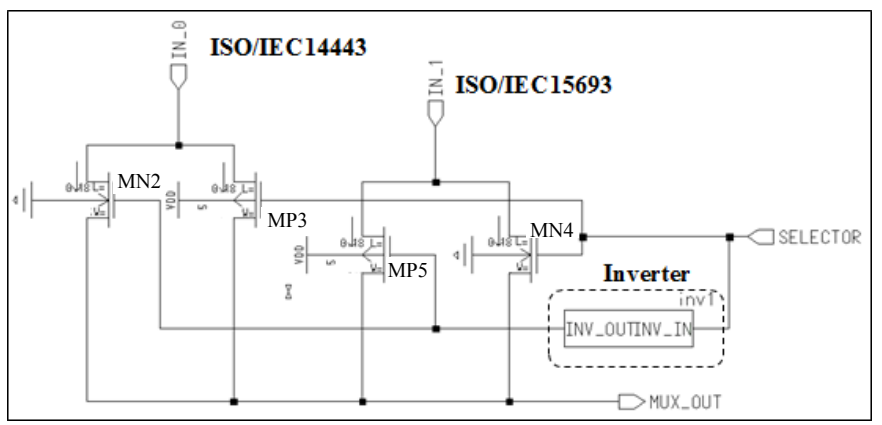

Fig. 7. 2-to-1 Multiplexer Circuit

\section{RESULTS AND DISCUSSIONS}

In order to test for the functionality of the proposed switch design, RF input signal with magnitude of $1.8 \mathrm{~V}$ and $3.3 \mathrm{~V}$ are used as the inputs to the design.

Figure 8 shows the simulated reference voltage over a temperature range of $-50^{\circ} \mathrm{C}$ to $150^{\circ} \mathrm{C}$. The variation in reference voltages for temperature below $-48^{\circ} \mathrm{C}$ are approximately $8.49 \mathrm{mV}$ and $4.67 \mathrm{mV}$ for $\mathrm{RF}$ input signal of $1.8 \mathrm{~V}$ and $3.3 \mathrm{~V}$ respectively. Since $\mathrm{V}_{\mathrm{REF}}$ is fixed over temperature range of $-48^{\circ} \mathrm{C}$ to $150^{\circ} \mathrm{C}$, a constant and temperature-stable reference voltage is obtained.

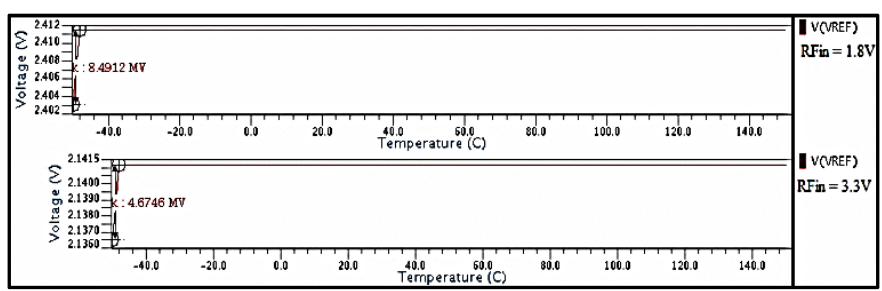

Fig. 8. Simulated temperature characteristics of output reference voltage
Figure 9 and Figure 10 show the simulated waveforms for the automated switch with RF input signal of $1.8 \mathrm{~V}$ and $3.3 \mathrm{~V}$ respectively. According to Figure 9, the $\mathrm{RF}_{\mathrm{IN}}$ signal of $1.8 \mathrm{~V}$ is fed into the envelope detector circuit and the peak of the $\mathrm{RF}_{\mathrm{IN}}$ signal is tracked as $\mathrm{V}_{\mathrm{ENV}}$ signal. Next, $\mathrm{V}_{\mathrm{ENV}}$ is applied into a two-stage op-amp circuit and the amplified signal is denoted as $\mathrm{V}_{\mathrm{IN}}$. The reference voltage $\mathrm{V}_{\mathrm{REF}}$ that is required in the comparator is generated by a CMOS voltage reference circuit. Since $\mathrm{V}_{\mathrm{IN}}(\sim 1.8 \mathrm{~V})$ is smaller than the $\mathrm{V}_{\mathrm{REF}}(\sim 2.644 \mathrm{~V})$, the output voltage represented by SELECTOR signal is at logic high state and fixed at $\mathrm{V}_{\mathrm{DD}}$ of $1.8 \mathrm{~V}$. At final stage, as SELECTOR signal is at logic high state, the IN_1 signal corresponded to ISO15693 standard is selected as MUX_OUT signal automatically.

On the contrary, referring to Figure 10 , since $\mathrm{V}_{\text {IN }}$ signal $(\sim 3.2957 \mathrm{~V})$ is greater than $\mathrm{V}_{\mathrm{REF}}$ signal $(\sim 2.644 \mathrm{~V})$, the output voltage represented by SELECTOR signal is at logic low state and stay at $0 \mathrm{~V}$. Therefore, at the final stage of switch design, as the SELECTOR signal is at logic low state, IN_0 signal that corresponding to ISO14443 standard is selected as MUX_OUT signal automatically.

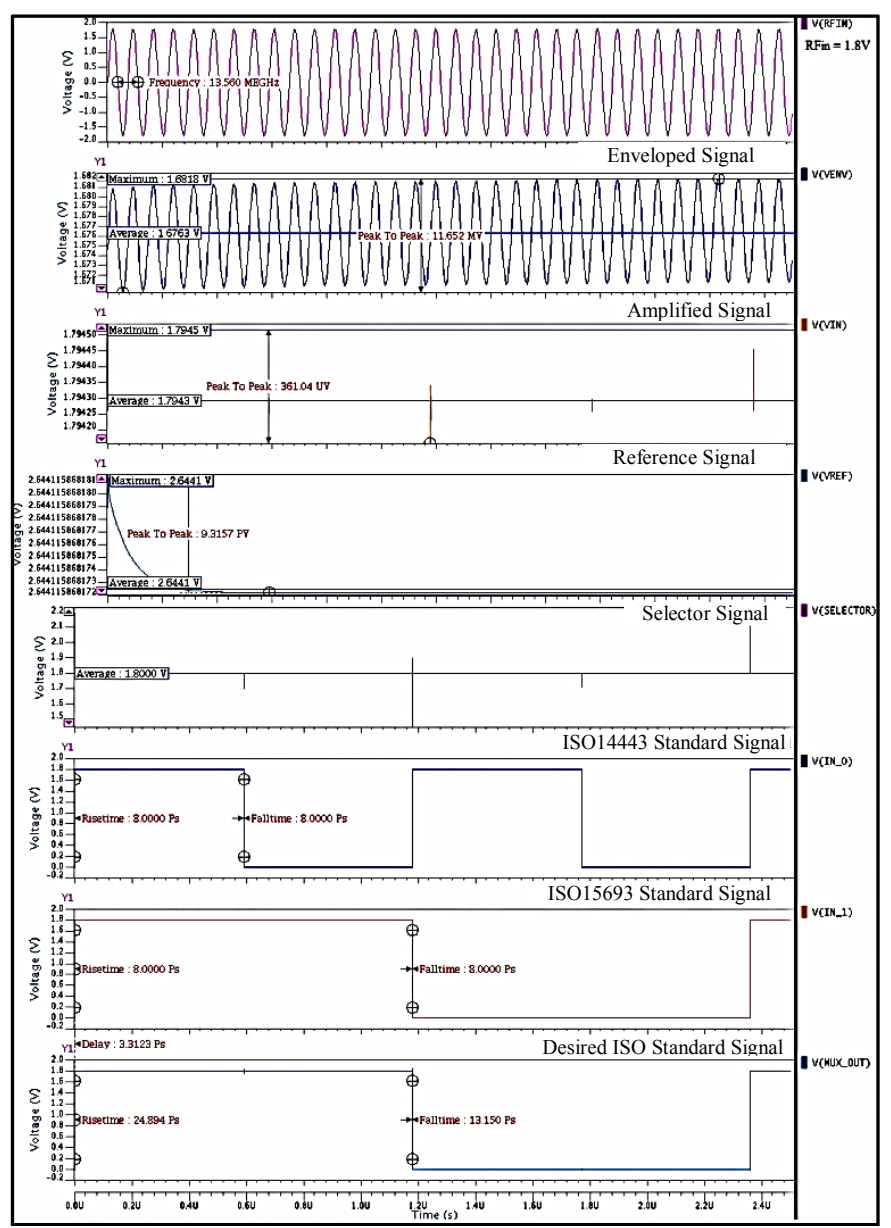

Fig. 9. Simulated input and output waveforms for automated switch design with RF input signal of $1.8 \mathrm{~V}$ 


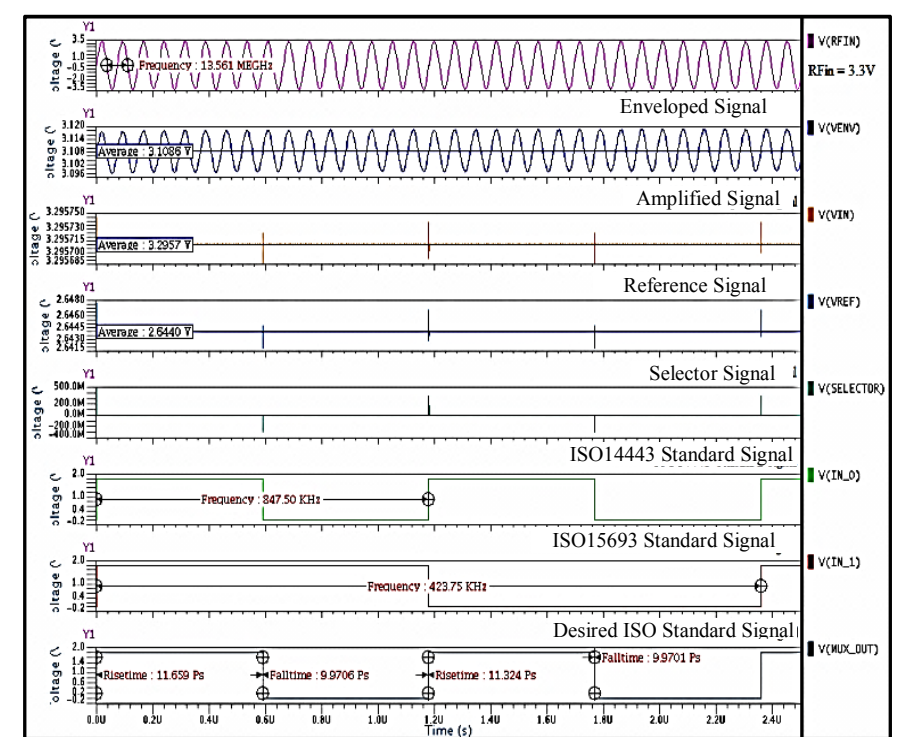

Fig. 10. Simulated input and output waveforms for automated switch design with RF input signal of $3.3 \mathrm{~V}$

\section{CONCLUSION}

The design of an automated switching mechanism for a multistandard $13.56 \mathrm{MHz}$ transponder has been presented. The proposed automated switch design conforms to the standards of ISO 14443 and ISO 15693 Single Subcarrier, working in HF of passive RFID transponder. The automated switch design was designed and validated using Mentor Graphics employing TSMC $0.18 \mu \mathrm{m}$ technology. Simulation results verified that the proposed switch design is capable of switching to the desired ISO standard accordingly in a multi-standard transponder protocol. Since this automated selection of the two different protocols are based on the incoming RF amplitudes, therefore it will work in most situations except when we are receiving weak signals from the reader to the vicinity card/transponder.

\section{REFERENCES}

[1] Y. F. Lim, M. K. Khaw and F. Mohd-Yasin, "A Multi-Standard Modulator for High Frequency Passive RFID Transponder", Circuits and Systems (MWSCAS), 2011 IEEE 54th International Midwest Symposium on, 23 September 2011.

[2] Y. H. Kim, Y. C. Choi, M. W. Seo, S. S. Yoo, and H. J. Yoo, “A CMOS Transceiver for a Multistandard13.56-MHz RFID Reader SoC”, IEEE Transactions on Industrial Electronics, vol. 57, No. 5, 5 May 2010.

[3] A. Shameli, A. Safarian, A. Rofougaran, M. Rofougaran and F. D. Flaviis, "An RFID System with Fully Integrated Transponder", University of California and Broadcom Corporation.

[4] C. Metzger, A. Ilic, P. Bourquin, F. Michahelles, E. Fleisch, "Distancesensitive High Frequency RFID Systems".

[5] N. M. Karim, "Design of an Integrated Demodulator for a $13.56 \mathrm{MHz}$ RFID reader", IEEE Symposium on Industrial Electronics and Applications, 4 October 2009

[6] P N. A. Fahsyar and N. Soin, "CMOS Implementation of Envelope Detector Circuit in $0.18 \mu \mathrm{m}$ Process", ICSE Proc. 2010

[7] P. K. Alli, "Testing a CMOS Operational Amplifier Circuit Using a combinational of Oscillation and $\mathrm{I}_{\mathrm{DDQ}}$ Test Methods.", Master thesis, Agriculture and Mechanical College, Louisiana State University, 2004.

[8] L. Najafizadeh, "Voltage References Using Mutual Compensation of Mobility and Threshold Voltage Temperature Effects", University of Alberta, Spring 2004.

[9] Jan M. Rabaey, A. Chandrakasan, and B. Nikolic, "Digital Integrated Circuits: A Design Perspective", 2nd Edition, Prentice Hall, 2003. 